\title{
Rome, Season Two Cast List
}

Lucius Vorenus

Titus Pullo

Atia of the Julii

Octavia of the Julii

Servilia of the Junii

Mark Antony

Octavian

Brutus

Cassius

Cleopatra

Charmian

Timon

Levi

Eirene

Gaia

Vorena the Elder

Cicero

Posca

Livia

Agrippa

Maecenas

Mascius

Newsreader

Erastes Fulmen

Jocasta

Duro

Caesarion

Ptolemy XIII

Julius Caesar

Niobe
Kevin McKidd

Ray Stevenson

Polly Walker

Kerry Condon

Lindsay Duncan

James Purefoy

Max Pirkis (episodes I 3 and I4) and Simon Woods

Tobias Menzies

Guy Henry

Lyndsey Marshal

Kathryn Hunter

Lee Boardman

Nigel Lindsay

Chiara Mastalli

Zuleikha Robinson

Coral Amiga

David Bamber

Nicholas Woodeson

Alice Henley

Allen Leech

Alex Wyndham

Michael Nardone

Ian McNeice

Lorcan Cranitch

Camilla Rutherford

Rafi Gavron

Max Baldry

Scott Chisolm

Ciarán Hinds

Indira Varma 